\title{
Desloratadine analysis: as a pharmaceutical preparation and after accelerating ageing
}

\author{
Katarzyna Bober ${ }^{* 1}$, Marlena PŁonka $^{2}$, Marek MiszczyK ${ }^{2}$ \\ ${ }^{1}$ Department of Analytical Chemistry, Faculty of Pharmacy, Medical University of Silesia in Katowice, Jagiellonska 4, 41-200 \\ Sosnowiec, Poland \\ ${ }^{2}$ Institute of Plant Protection - National Research Institute, Pesticide Quality Testing Laboratory, Gliwicka 29, 44-153 Sosnicowice, Poland
}

\section{ARTICLE INFO \\ Received 08 January 2015 \\ Accepted 15 September 2015}

\section{Keywords:}

desloratadine,

densitometry,

HPTLC,

GC-MS,

accelerating ageing.

\begin{abstract}
Desloratadine is a biologically active compound that is not described in the Polish Pharmacopoeia IX, hence, its study is particular important. The aim of this work was to establish a procedure for desloratadine analysis by way of HPTLC in combination with densitometry, so as to be able to ascertain its presence and degree of presence within pharmaceutical preparations. In our work, a mixture of ethyl acetate, n-butanol, ammonia and methanol was used as the mobile phase. Moreover, HPTLC plates precoated with silica gel $60 \mathrm{~F}_{254}$ were also employed. The proposed method was tested and subsequently validated. Spectrodensitometric analysis was then performed to determine the optimal wavelength for the quantitative determination $(\lambda=276 \mathrm{~nm})$, and following this, a quantitative analysis of desloratadine within certain pharmaceutical preparations was performed. Our research also took into consideration an analysis of the products of desloratadine decomposition that come about as a result of the accelerated aging of its solutions. The employed procedure for accelerating the aging of such desloratadine solutions consisted of heating these at $40^{\circ} \mathrm{C}$ and then irradiating the solution surfaces with UV light. The changing color of these solutions after 2 hours of exposure served to indicate that degradation had occurred. Of note: as a result of irradiation with UV light, desloratadine content was seen to decrease with time, declining to almost zero after 30 hours. However, heating a solution of desloratadine alone did not induce a change in its content. Solutions of desloratadine that had previously undergone irradiation and heating were also analyzed to ascertain whether new substances were present. For this purpose, the GC-MS process was employed. As a result of this procedure, the spectrum of the solution after aging showed the presence of several new peaks that displayed retention several times larger and smaller than the normal desloratadine peak.
\end{abstract}

\section{INTRODUCTION}

Antihistamine preparations are commonly sold items in Poland's pharmacies. Desloratadine is a substance with antihistamine activity, and it is a ingredient found within several very popular recent pharmaceutical preparations. Polish Farmacopeia IX, however, does not describe any recommended method of desloratadine analysis [8]. Standard approaches for desloratadine analysis as described in literature are liquid chromatography combined with mass spectrometry $[2,4,9,12,13]$, spectrometry, spectrofluorimetry and high performance liquid chromatography $[1,2,3,7,10,14]$.

\footnotetext{
* Corresponding author

e-mail: e-mail: bober@sum.edu.pl

tel.: + 48323641560, fax +48323641543
}

The aim of the work was to set out a procedure for desloratadine analysis utilizing high performance thin layer chromatography combined with densitometry, as well to establish the effect of accelerated ageing upon the contents of solutions of desloratadine.

\section{MATERIALS AND METHOD}

\section{Thin layer chromatography}

A standard solution of desloratadine (Sigma-Aldrich, USA) was prepared in ethanol (POCh, Poland) with concentration $1 \mathrm{mg} / 1 \mathrm{~mL}$. In addition, particular dilutions of desloratadine standard solution were made up for the purpose of obtaining a standard curve. HPTLC plates pre-coated 
with silica gel (\#1.05548, Merck, Germany) were used for analysis. The plates were activated first at a temperature of $120^{\circ} \mathrm{C}$ for $30 \mathrm{~min}$. These plates were then developed to the height of $7.5 \mathrm{~cm}$ at room temperature. The glass chromatographic chamber was first saturated with vapour of mobile phase (a mixture of ethyl acetate - n-butanol - 25\% ammonia - methanol in volume ratio 30:7:6:7) for $30 \mathrm{~min}$. The desloratadine solution were then spotted using a glass micro capillary (Camag, Switzerland). The composition of the eluents was chosen experimentally.

\section{Spectrodensitometric analysis}

Spectrodensitometric analysis by way of the use of a Camag TLC 3 densitometer was undertaken for the purpose of establishing the maximum wavelength for the quantitative analysis of desloratadine. This analysis was led in the range of wavelengths between 200 and $400 \mathrm{~nm}$. The slit dimension was $8.00 \times 0.40 \mathrm{~nm}$, while the scanning speed was 100 $\mathrm{nm} / \mathrm{s}$. Scanning was performed as absorption. The radiation sources employed in this experiment were deuterium and wolfram lamps.

\section{Quantitative densitometric analysis}

Densitometric scanning was performed by way of using a Camag TLC 3 densitometer and the winCATS software. The produced chromatographic plates were scanned using wavelengths settled previously. Deuterium and wolfram lamps were used as radiation sources.

\section{Method validation}

Method validation for desloratadine analysis was performed according to $\mathrm{ICH}$ recommendations $[6,11]$. The linearity range, limit of detection (LOD), limit of quantification (LOQ), as well as precision and accuracy were determined for the proposed method. The linearity range was established on the basis of a previously generated standard curve. All spotted solutions ( $3 \mu \mathrm{l}$ spots) were in a range between 3.00 and $0.05 \mu \mathrm{g} / \mathrm{spot}$. The mobile phase was a mixture of ethyl acetate - n-butanol $-25 \%$ ammonia - methanol in a volume ratio of 30:7:6:7. The analysis was done in triplicate. The standard curve was prepared as a relationship between area of densitometric peak [AU] and desloratadine content $[\mu \mathrm{g} / \mathrm{spot}]$.

Limits of detection (LOD) and quantification (LOQ) was calculated on the base of data obtained from the calibration curve.

The accuracy of the analysis was ascertained by calculating the percentage of the recovery. For this purpose, the method of standard additions was employed. The analysis was performed six times. The precision of the method was determined on the basis of densitometric measurements, and expressed as the value of the relative standard deviation (RSD [\%]).

\section{Analysis of desloratadine in particular pharmaceutical preparations}

Using chromatographic conditions settled previously, a quantitative analysis was undertaken of desloratadine content in certain pharmaceutical preparations (Azomyr Polpharma, Poland and Aerius - Schering-Plough, Poland).
Tablets were first ground in a mortar and placed into a flask. Ethyl alcohol was added and then the flask was shaken for 30 minutes. The solution was subsequently filtered through Whitmann filter pape, and the volume was made up to $10 \mathrm{~mL}$ to give a solution containing $1 \mathrm{mg}$ of desloratadine per $1 \mathrm{~mL}$ of solution. The filtrate was subsequently analysed.

\section{Accelerating ageing}

The procedure for accelerating ageing involved exposing desloratadine solutions to UV $(1=254 \mathrm{~nm})$ light and temperatures of $40^{\circ} \mathrm{C}$. Desloratadine analysis during this phase of the experiment was carried out in the chromatographic conditions used previously for the analysis of standard solution and for the investigated pharmaceutical preparations (HPTLC plates (\#1.05548, Merck, Germany) and mobile phase ethyl acetate - n-butanol - 25\% ammonia - methanol at a volume ratio 30:7:6:7). Quantitative densitometric analysis was led using wavelength $1=276 \mathrm{~nm}$, by way of using a Camag TLC 3 densitometer and software winCATS.

\section{GC - MS analysis}

Initial solutions of desloratadine and solutions previously exposed to UV light and heating, were analysed by way of employing GC - MS Gas chromatography (Agilent 7890A Series, with mass spectrometer detector Agilent 5975C (EIelectron impact) and Headspace Sampler G1888, as well as ChemStation instrument control data acquisition and data evaluation).

\section{RESULTS AND DISCUSSION}

The values characterizing the employed method of desloratadine analysis are presented in Table 1. Data are sourced from the obtained calibration curve.

Table 1. Data characterizing the calibration curve and the method of desloratadine analysis by use of high performance thin layer chromatography.

\begin{tabular}{|l|c|}
\hline linearity range $[\mu \mathrm{g} / \mathrm{spot}]$ & $3 \div 0.4026$ \\
\hline intercept & $2312.2400( \pm 79.5967)$ \\
\hline slope & $1773.0400( \pm 125.1260)$ \\
\hline standard deviation $\sigma$ & 207.3230 \\
\hline determination coefficient $\mathrm{R}^{2}[\%]$ & 99.06 \\
\hline LOD $[\mu \mathrm{g} / \mathrm{spot}]$ & 0.2959 \\
\hline LOQ $[\mu \mathrm{g} / \mathrm{spot}]$ & 0.8966 \\
\hline \multirow{2}{*}{ accuracy (\% of recovery) } & 100.21 (Aerius) \\
\hline precision RSD [\%] & 96.57 (Azomyr) \\
& 2.70 (Aerius) \\
\hline
\end{tabular}

Figure 1 presents the ascertained spectrodensitogram of desloratadine, whilst Figure 2 is the densitogram of the desloratadine standard solution established by way of using the wavelength previously settled upon.

Densitograms of desloratadine in the analyzed drugs items were seen to be identical to the densitogram for a standard solution of desloratadine. RF value for all analysis was 0.53 .

As a result of accelerating ageing, a change of colour of solution (from colourless to yellowish) was observed after 2 hours of heating and exposure to UV light. This evidences the degradation process affecting the investigated compound. 
Due to exposure to UV light, the contents of the biologically active compound (desloratadine) was established as decreasing with time. After 30 hours, the aforementioned declined reached to almost zero $(0.035 \mathrm{mg} / \mathrm{spot})$, whilst the initial contents was $3 \mathrm{mg} / \mathrm{spot}$. Heating the desloratadine solution alone did not induce a change in content of this compound. Indeed, after 30 hours of heating, the contents of desloratadine was still about $2.940 \mathrm{mg} / \mathrm{spot}$. The following figures present the densitograms of desloratadine at the initial point of analysis (Fig.2), after 30 hours of exposure to UV light (Fig.3) and after 30 hours of heating (Fig.4). The subsequent figure (Fig.5) is of the desloratadine content of investigated solutions after exposure to UV light and heating.

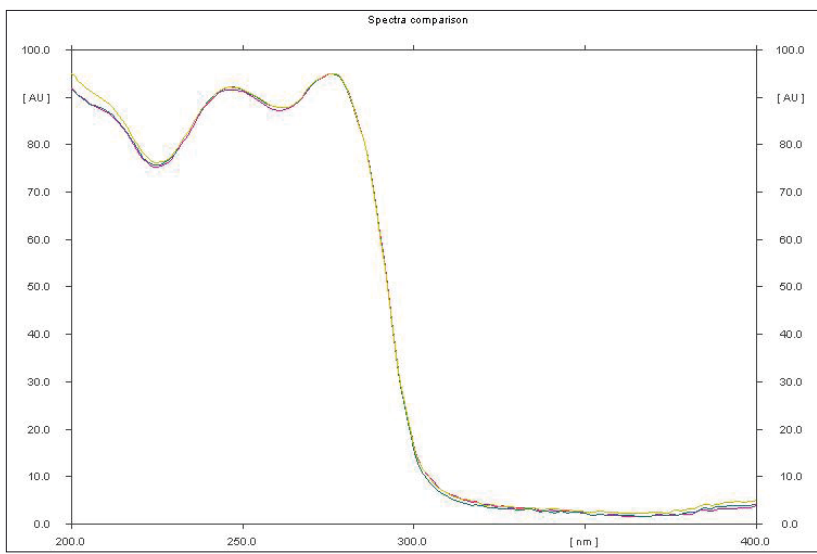

Figure 1. Spectrodensitogram of desloratadine

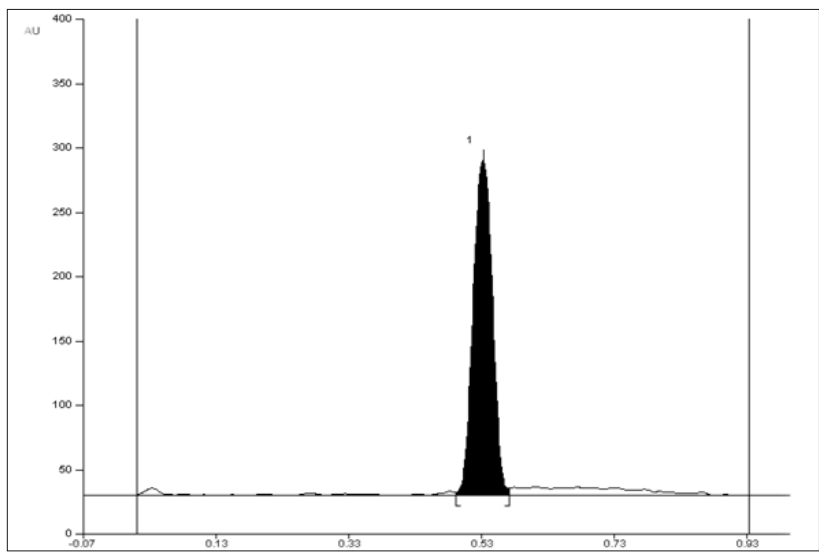

Figure 2. Densitogram of desloratadine, $\lambda=276 \mathrm{~nm}$

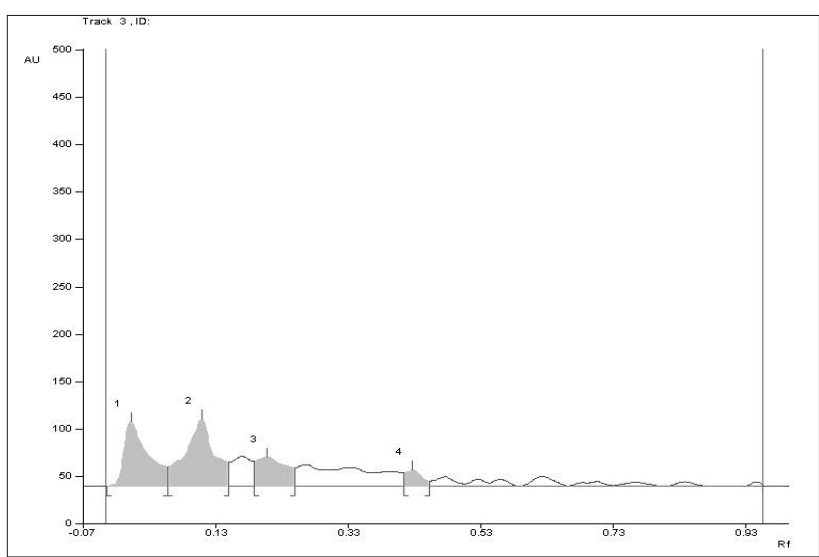

Figure 3. Densitogram of desloratadine after $30 \mathrm{~h}$ of exposure to UV light, $\lambda=254 \mathrm{~nm}$
Figure 3 reveals the existence of new densitogram peaks. This indicates the presence of products that had come about due to exposure to UV light. Only one clear peak is seen in Figure 4. This that of desloratadine (the other peak seen $(\mathrm{RF}=1)$ is in actuality, the face of the mobile phase). Figure 5 clearly indicates changes in desloratadine content as a result of exposure to UV light.

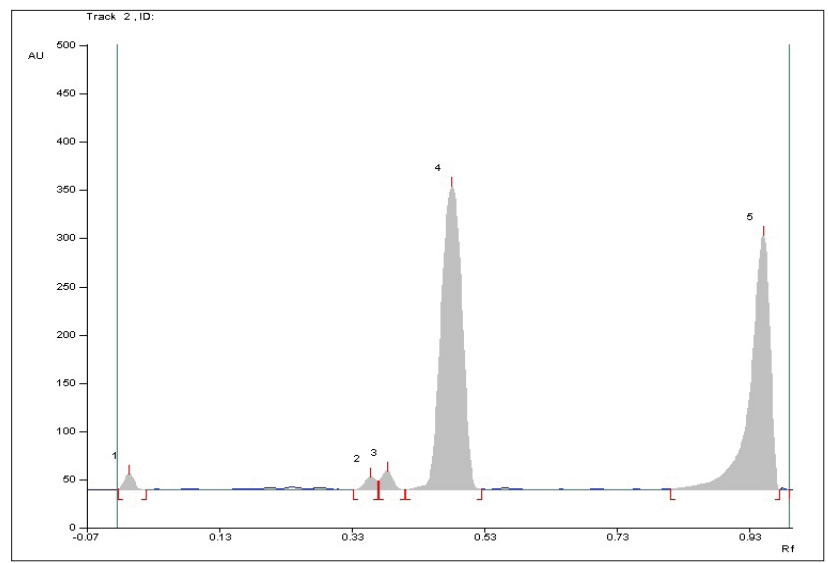

Figure 4. Densitogram of desloratadine after $30 \mathrm{~h}$ of heating to a temperature of $40^{\circ} \mathrm{C}$

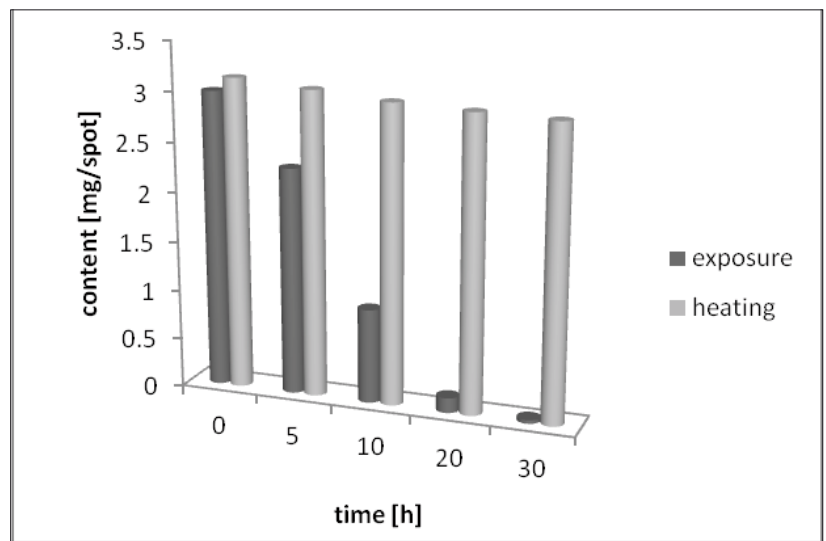

Figure 5. Changes in content of desloratadine following exposure to UV light and heating

Figures 6 and 7 are comparisons of desloratadine spectrum GC - MS in initial solution and in solution after 30 hours of exposure to UV light, $\lambda=254 \mathrm{~nm}$ (Fig.6), as well as after 30 hours of heating at a temperature of $40^{\circ} \mathrm{C}$ (Fig.7).

In the initial solution, we found some loratadine contamination. This was identified as impurity E (Imp E) [5]. Note: the presence of this chemical is not evident when analysis is done by using TLC with densitometry. In our work, other compounds were created as a result of heating, but analysis revealed that loratadine content had decreased. GC - MS analysis confirmed the results of the HPTLC - densitometry analysis. Furthermore, it was evident that heating did not have influence on desloratadine content. However, GC - MS analysis also confirmed that desloratadine content significantly decreases as a result of exposure to UV light. The obtained spectrum indicates as well, that as a result of exposure to UV light, many new compounds were created. These are products of the degradation of desloratadine and products of the degradation of the impurities present in the initial solution. 


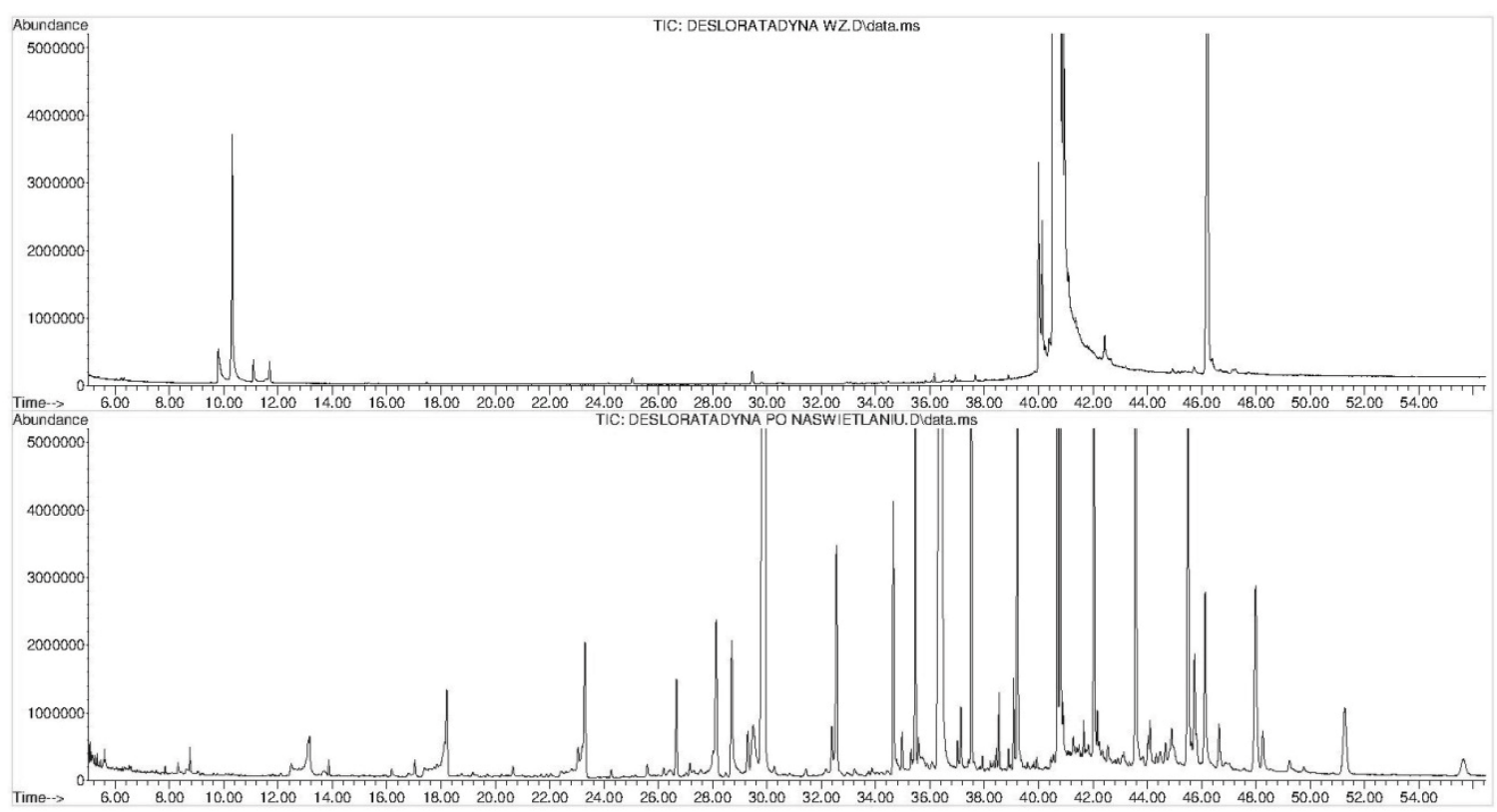

Figure 6. Spectrum GC - MS for a standard solution of desloratadine and for a solution after 30 hours of exposure to UV light

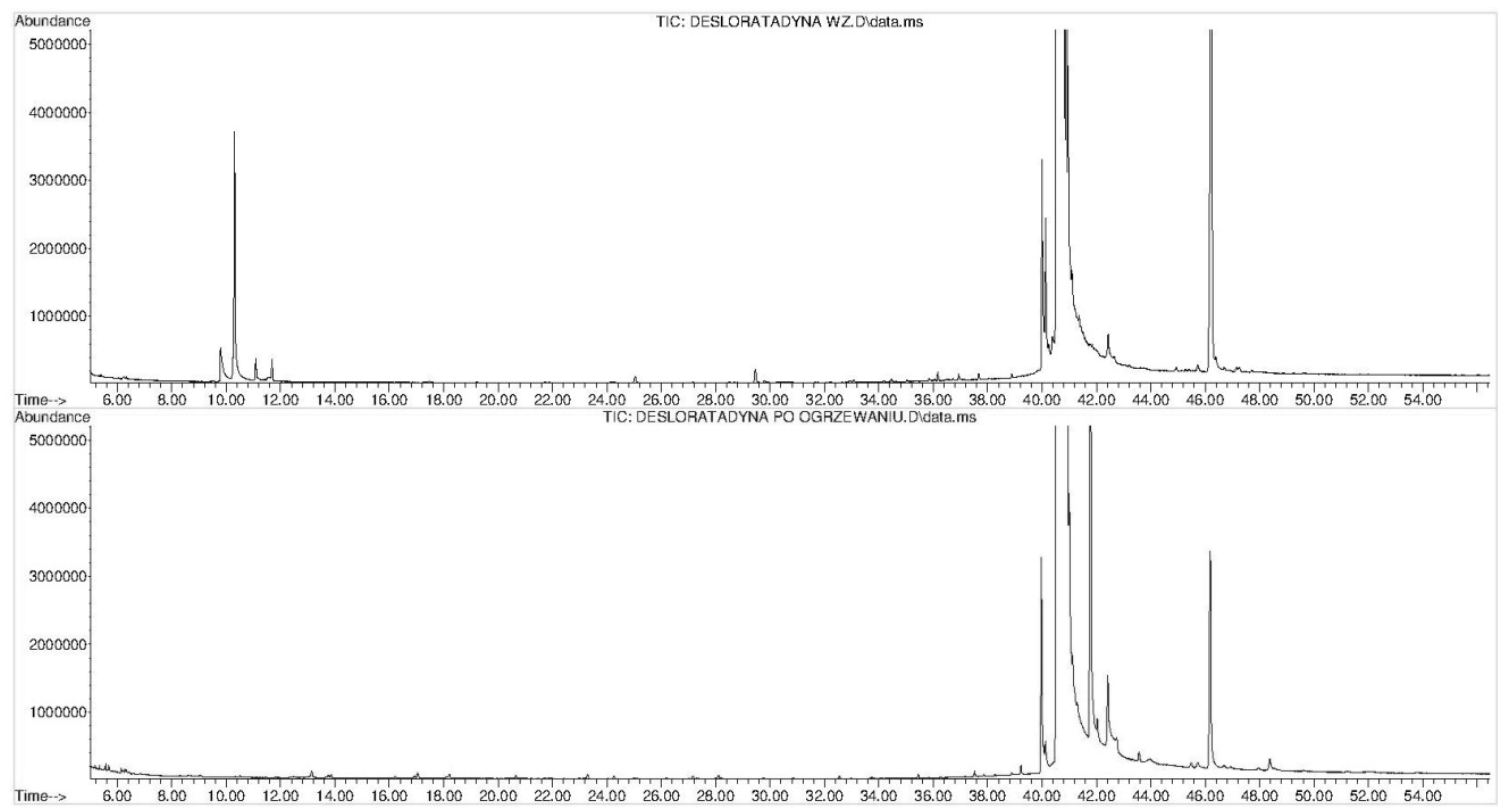

Figure 7. Spectrum GC-MS for a standard solution of desloratadine and for a solution after 30 hours of heating

\section{ACKNOWLEGMENT}

This research was financed by the Medical University of Silesia as part of statutory research project KNW-1-013/N/3/0 and KNW-1-006/N/4/0.

\section{REFERENCES}

1. Caglar S., Oztunc A.: A sensitive spectrophotometric determination of desloratadine in tablets. J. AOAC Int., 90(2), 372, 2007.

2. Caglar S., Toker S.E.: Simultaneous determination of desloratadine and pseudoephedrine sulphate in tablets by high performance liquid chromatography and derivative spectrophotometry. Rev. Anal. Chem. 30, 145, 2011.

3. El-Enany N.M., El-Sherbiny D., Belal F.: Spectrophotometric, spectrofluorometric and HPLC determination of desloratadine in dosage form and human plasma. Chem. Pharm. Bull., 15(12), 1662, 2007.
4. Hasnain M.S. et al.: Development and validation of an improved LC-MS/MS method for the quantification of desloratadine and its metabolite in human plasma using deuterated desloratadine as internal standard. J. Pharm. BioAllied Sci. 5(1), 74, 2013.

5. http://www.tlcpharmachem.com/tlc_item.php?upc $=\mathrm{L}-1027 \& \mathrm{li}=$ le $\_21 \&$ sub $=\mathrm{L}$

6. ICH harmonized tripartite guideline: validation of analytical procedures: text and methodology, Q2 (R1), Geneva, 2005, http:// www.ich.org Accessed 30 August 2013.

7. Mohamed G.G., Abou Attia F.M., Ibrahim N.S.: Development and validation of spectrophotometric and HPLC methods for the determination of desloratadine in tablets and syrup. J. Pharm. Res. 5(5), 2799, 2012.

8. Polish Farmacopeia IX, Polish Pharmaceutical Society, Warsaw, 2011. 
9. Ponnuru V.S, Challa B.R., Nadendla R.: Quantification of desloratadine in human plasma by LC-ESI-MS/MS and application to a pharmacokinetic study. J. Pharm. Anal. 2(3), 180, 2012.

10. Rele R.V., Gurav P.J.: A Simple extractive spectrophotometric determination of loratadine, desloratadine and rupatadine from pharmaceutical formulations. Int. J. Pharma Bio Sci. 3(2), 89, 2012.

11. Renger B., Vegh Z., Ferenczi-Fodor K.: Validation of thin layer and high performance thin layer chromatographic methods. J. Chromatogr. A, 1218, 2712, 2011.
12. Srinubabu G. et al.: Development and validation of high-throughput liquid chromatography-tandem mass spectrometric method for simultaneous quantification of loratadine and desloratadine in human plasma. J. Chromatogr. B, 860(2), 202, 2007.

13. Vlase L. et al.: Determination of loratadine and its active metabolite in human plasma by high- performance liquid chromatography with mass spectrometry detection. J. Pharm. Biomed. Anal. 44(3), 652, 2007.

14. Yang L.Y., Rudewicz P.J.: Application of new liquid chromatography - tandem mass spectrometry technologies for drug development support. J. Chromatogr. A, 926(1), 43, 2001. 